\title{
Blind Adaptive Equalization for Aerospace Communication Channels
}

\author{
Elessaid S. Saad ${ }^{*}$ \\ *Department of Electrical Engineering, Karary University, Khartoum,12304, Sudan \\ §elessaid@karary.edu.sd,
}

\begin{abstract}
In some communication systems, it is desirable for the receiver to synchronize to the received signal and to adjust the equalizer without having knowledge of a training sequence. Blind equalization uses the initial adjustment of the coefficients without making use of a training sequence. Different adaptive blind equalization algorithms have been developed over the past four decades. In this paper, we investigate the effect of blind equalization on space communication channels. The space channel under investigation is considered to be a multipath frequency selective channel having four paths. The channel is subjected to the phenomenon of Inter Symbol Interference (ISI) which severely degrades the performance of the space communication system. Two blind algorithms are used in equalizer adjustment. The impulse responses of the space channel, the blind equalizer and the combination of channel and equalizer for QPSK and 16-QAM transmission are shown. The scatter diagrams for the transmitted sequence, received sequence, and the output of the equalizer using two of the blind algorithms are shown.
\end{abstract}

Key words-aerospace; multipath; ISI; blind; equalization

\section{INTRODUCTION}

Aerospace multipath fading channels are characterize is in terms of their multipath spread, Doppler spread, and the total bandwidth at bandpass available for signal transmission [1], [2]. It is required for the designer to choose suitable pulse duration so that the effect of ISI can be reduced.

It is also required to determine the type of fading encountered by the signal, and discuss the possibility of use of appropriate frequency diversity. Modeling aerospace channel as multipath channel has been discussed widely in the literature [2], [3]. In this work, the aerospace multipath fading channel under consideration is assumed to be have a response of the form:

$$
h(n)=\alpha_{1} \delta(n)+\alpha_{2} \delta(n-1)+\alpha_{3} \delta(n-2)-\alpha_{4} \delta(n-3),
$$

The objective of this work is to design adaptive equalizers for the channel based on two blind algorithms. The blind equalizer structure is shown in Fig. 1.

One could observe that the scatter diagram at the output of the equalizer is more focused when compared with that of the CMA2-2.

The transmitted symbols $s(i)$ through the channel are corrupted by additive complex-valued white noise $\mathrm{n}(\mathrm{i})$. The received signal is processed by a linear equalizer the output of which is

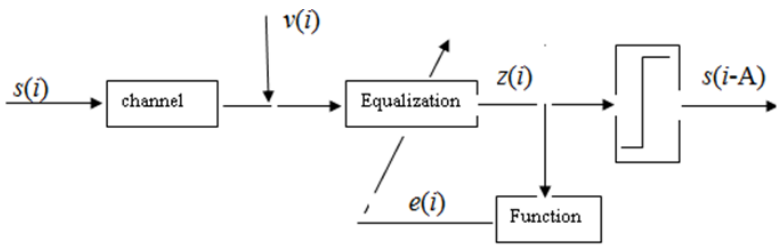

Fig. 1. General blind adaptive equalizer $\mathrm{z}$ (i) are fed into a decision device to obtain $\hat{s}(i-\Delta)$. In this proposed work, the equalizer is supposed to operate blindly, i.e., without a reference sequence and hence without a training mode . Most of blind equalizers use the output of the equalizer, $z(i)$, to generate the error signal $e(i)$, which is used to adapt the equalizer coefficients according to the rule:

$$
w_{i}=w_{(i-1)}+\mu u_{i}^{*} e(i),
$$

where $w$ is the regressor at time $i$. Some blind algorithms use the output of the decision device, to evaluate the error signal $e(i)$.
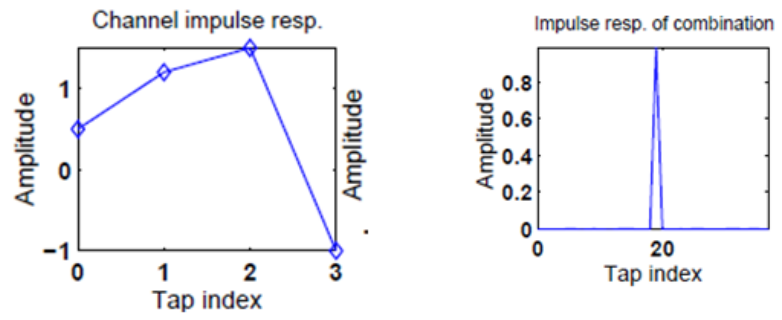

Equalizer impulse resp.

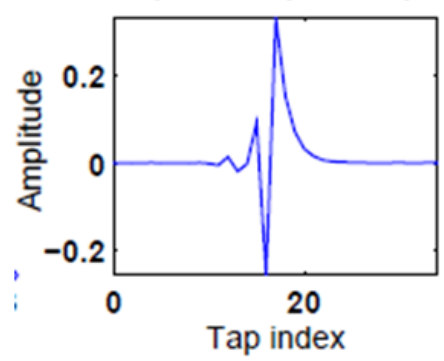

Fig. 2. Channel impulse responses for QPSK 

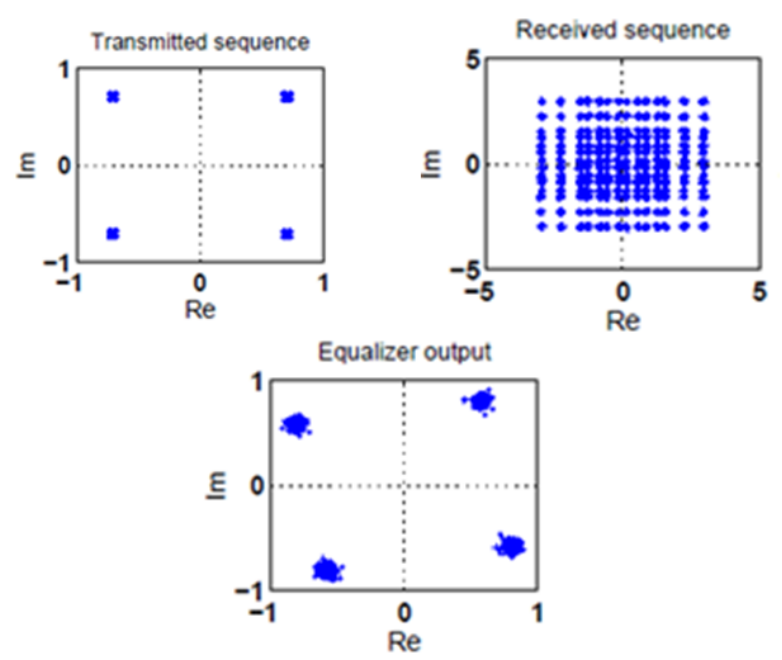

Fig. 3. Scatter diagrams for QPSK - CMA2-2
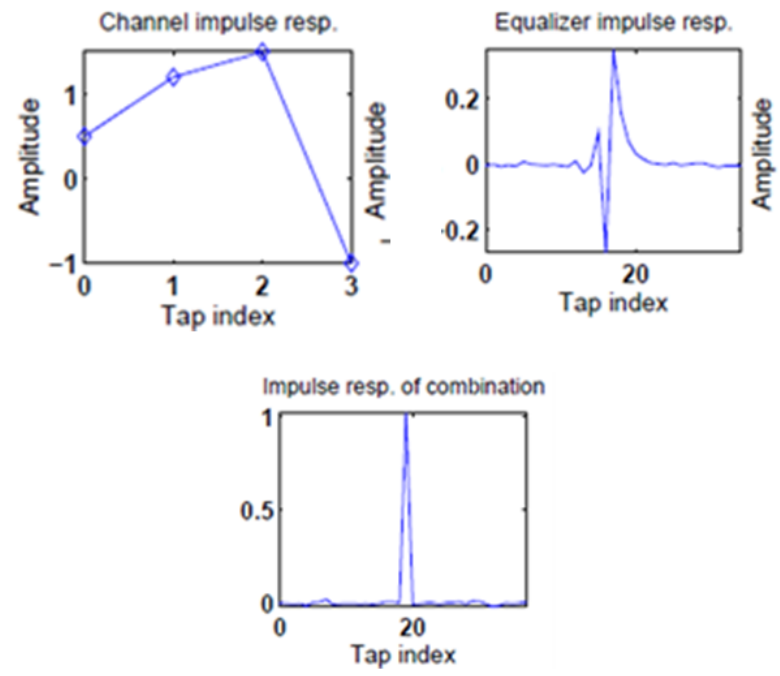

Fig. 4. Impulse responses for 16-QAM.
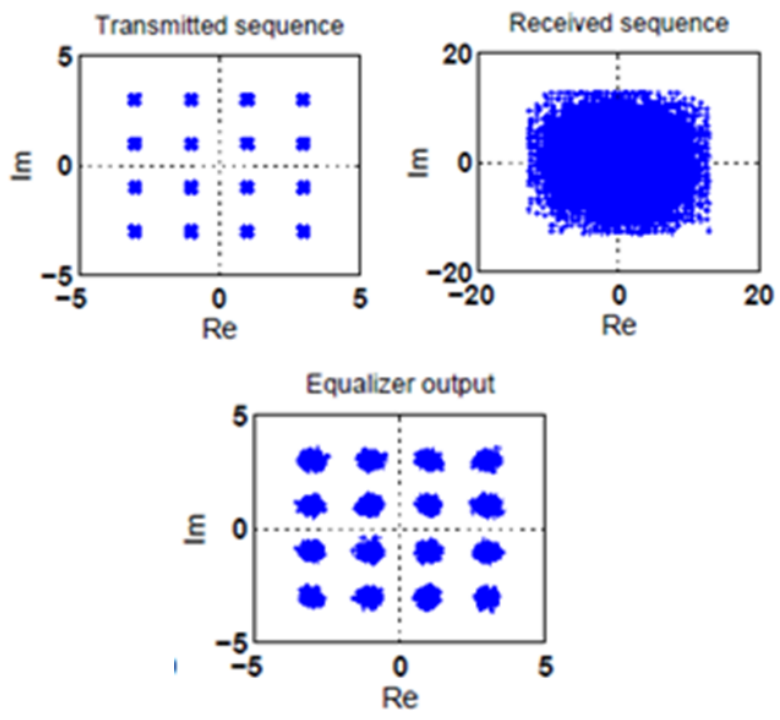

Fig. 5. Scatter diagrams using CMA2-2.
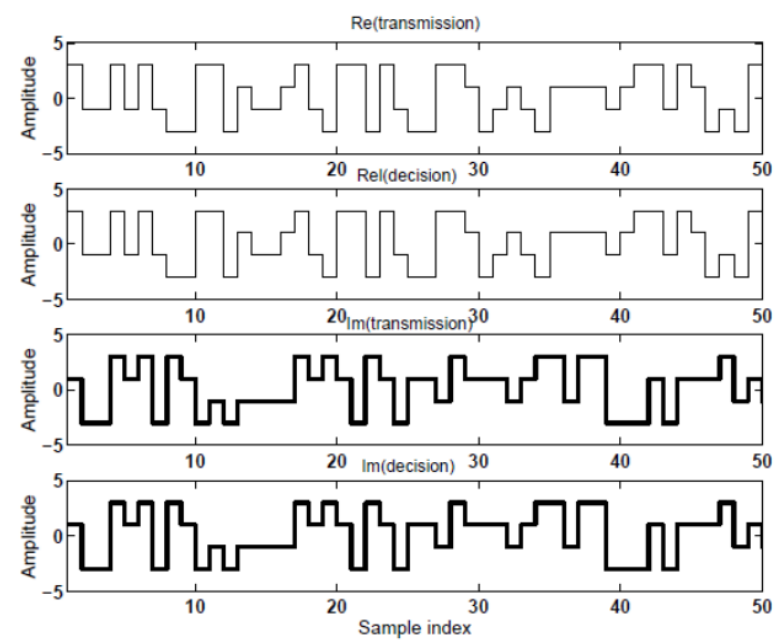

Fig. 6. Time diagrams of 50 transmitted.

\section{BLIND ALGORITHMS}

Constant Modulus Algorithm CMA1-2 and NCMA: The first blind algorithm is the constant modulus algorithm $C M A 1-2$ which can be summarized as follows: Given a zeromean random vector $\mathbf{u}$ with realizations and positive scalar $\left[u_{0}, u_{1}, ..\right]$ [4], [5]. An optimal weight vector $\mathbf{w}^{o}$ that solve

$$
\min _{\mathbf{w}}=E(\gamma-|\mathbf{u w}|)^{2},
$$

can be approximated iteratively via the recursion

$$
\left.w_{i}=w_{(} i-1\right)+\mu u_{i}^{*}\left[\gamma \frac{z(i)}{|z(i)|}-z(i)\right], z(i)=\mu_{i} w_{(i-1)}, i \geqq 0,
$$

where $\mu$ is a positive step-size (usually small). The recursion can also be implemented in normalized form as (NCMA)

$w_{i}=w_{(i-1)}+\frac{\mu u_{i}^{*}}{\left\|\mu_{i}\right\|^{2}}\left[\gamma \frac{z(i)}{|z(i)|}-z(i)\right], z(i)=\mu_{i} w_{(i-1)}, i \geqq 0$,

in both cases, we set $\left.w_{i}=w_{(} i-1\right)$ when $z(i)=0$

Constant Modulus Algorithm CMA2-2: The second blind algorithm is the constant modulus algorithm CMA2-2 which can be summarized as follows: Given a zero-mean random vector $\mathbf{u}$ with realizations $\left[u_{0}, u_{1}, ..\right]$ and positive scalar $\gamma$. An optimal weight vector $\mathbf{w}^{o}$ that solve

$$
\min _{\mathbf{w}}=E\left(\gamma-|\mathbf{u w}|^{2}\right)^{2},
$$

can be approximated iteratively via the recursion

$\left.w_{i}=w_{(} i-1\right)+\mu u_{i}^{*} z(i)\left[\gamma-|z(i)|^{2}\right], z(i)=\mu_{i} w_{(i-1)}, i \geqq 0$,

where $\mu$ is a positive step-size (usually small).

Multi-Modulus Algorithm MMA: The Multi-Modulus Algorithm MMA can be summarized as follows: Given a zeromean random vector $\mathbf{u}$ with realizations $\left[u_{0}, u_{1}, ..\right]$ and positive scalar [6], [7]. An optimal weight vector $\mathbf{w}^{o}$ that solve

$$
\min _{w}=E\left\{\left[(\operatorname{Re}(\mathbf{u w}))^{2}-\gamma\right]^{2}+\left[(\operatorname{Im}(\mathbf{u w}))^{2}-\gamma\right]^{2}\right\},
$$



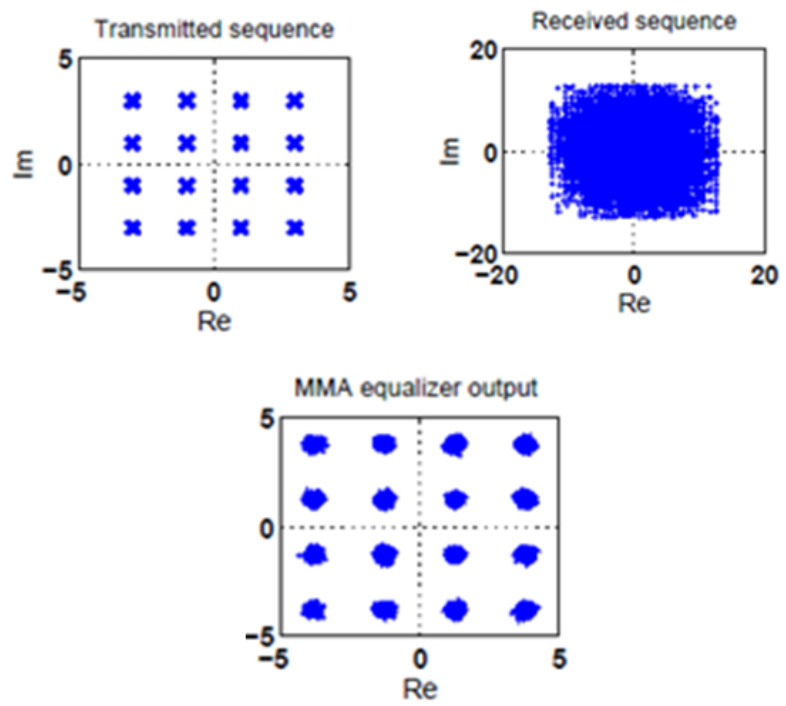

Fig. 7. Scatter diagrams using MMA.

can be approximated iteratively via the recursion

$$
\left\{\begin{array}{c}
\left(z(i)=u_{i} w_{(i-1)}\right. \\
a(i)=\operatorname{Re}[z(i)] \\
b(i)=\operatorname{Im}[z(i)] \\
e(i)=a(i)\left[\gamma-a^{2}(i)\right]+j b(i)\left[\gamma-b^{2}(i)\right] \\
w_{i}=w_{(}(i-1)+\mu u_{i}^{*} e(i)
\end{array}\right\}
$$

where $\mu$ is a positive step-size (usually small).

\section{SIMULATION}

First, a 35 tap equalizer is trained by 104 QPSK symbols using CMA2-2. The value of $\gamma$ is chosen as $\frac{E|s|^{4}}{E|s|^{2}}$ which is in turns of the second and fourth-order moments of the symbol constellation. For QPSK, data is 1. The signal to noise ratio level at the input of the equalizer is set to 30 $\mathrm{dB}$ and $\mu$ to 0.001 . The impulse response of the channel, the equalizer, and the combined channel-equalizer are plotted at the end of the adaptation process. The scatter diagrams of the transmitted sequence, the received sequence, and the sequence at the output of the equalizer are also plotted. Second, the simulation is repeated using $3 \times 104$ data symbols of $16-\mathrm{QAM}$, for which $\gamma=13.2$ and $\mu=0.000001$. In a third experiment, the simulation in the second part is repeated using MMA.

\section{RESULT AND DISCUSION}

Two plots are generated from simulation in the first part. Figure 2 shows the impulse responses of the channel, the equalizer, and the combination channel-equalizer. Observe that the latter has a peak at sample 19 so that the delay introduced by the channel and equalizer is 2 . Figure 3 shows scatter diagrams of the transmitted sequence, received sequence, and the output of the equalizer for QPSK transmissions and using CMA2-2. The first 2000 samples are ignored in order to give the equalizer sufficient time for convergence. Three plots generated from the second part of the simulation. Figure 4 shows the impulse responses of the channel, the equalizer, and the combination channel-equalizer.

Figure 5 shows a typical plot of the scatter diagrams at transmission, reception, and output of the equalizer for 16-QAM transmissions. It can be seen that although symbols from 16 QAM do not have constant modulus, CMA2-2 still functions properly; even though more slowly.

Figure 6 shows the real and imaginary parts of 50 transmitted symbols and the corresponding 50 decisions by the slicer (with the delay of 19 samples accounted for in order to synchronize the signals). These 50 symbols are chosen from the later part of the data after the equalizer has converged. Three plots are generated from simulation in third experiment. Here we only show in Fig. 7 the resulting scatter diagrams for MMA. The first 15000 samples are ignored in order to give the equalizer sufficient time for convergence.

\section{CONCLUSION}

An aerospace communication channel is modeled as a multipath fading channel subjected to ISI. Adaptive equalizer is designed based on two blind algorithms. The combined response of the channel and equalizer is obtained using two blind algorithms. Scatter diagrams are also obtained for QPSK and QAM Constellations. The two algorithms perform well, with the MMA outperforming the CMA2-2 in focusing.

\section{ACKNOWLEDGMENT}

This work was partially supported by Karary University.

\section{REFERENCES}

[1] P.A. Bello, "Aeronautical channel characterization," IEEE Trans. Commun., vol. COM-21, pp. 548-563, May 1973.

[2] Qiang Lei; Micael Rice Multipath Channel Model for Over-Water Aeronautical Telemetry IEEE Transactions on Aerospace and Electronic Systems (Volume: 45, Issue: 2, April.

[3] M. Rice A. Davis A multipath channel model for wideband aeronautical telemetry IEEE Milcom 2002 proceedings.

[4] Masoud Salehi John G. Proakis Digital Communications 2008 McGrawHill

[5] Hatzinakos, D., and Nikias, C. L. (1991). Blind Equalization Using a Tricepstrum-Based Algorithm, IEEE Trans. Commun, vol. COM-39, pp. 669682, May.

[6] Ding, Z. (1990). Application Aspects of Blind Adaptive Equalizers in QAM Data Communications, Ph.D. Thesis, Department of Electrical Engineering, Cornell University.

[7] Ali H Sayed. Fundamentals of Adaptive Filtering 2003, Wiley and Sons 